\title{
Investigação de Suspeita de Abuso Sexual Infantojuvenil: O Protocolo NICHD
}

\author{
Lúcia Cavalcanti de Albuquerque Williams ${ }^{1}$ \\ Chayene Hackbarth \\ Departamento de Psicologia da Universidade Federal de São Carlos, São Carlos, \\ São Paulo, Brasil \\ Carlos Aznar Blefari \\ Maria da Graça Saldanha Padilha \\ Universidade Tuiuti do Paraná, Curitiba, Paraná, Brasil \\ Carlos Eduardo Peixoto \\ Instituto Nacional de Medicina Legal e Ciências Forenses, Porto, \\ Distrito do Porto, Portugal
}

\section{Resumo}

O presente artigo consiste em uma revisão sistemática de literatura que se refere à escuta adequada e às estratégias para investigação da violência sexual infantil, com especial ênfase no Protocolo NICHD (National Institute of Child Health and Human Development), reconhecido pela literatura internacional especializada como um dos instrumentos mais adequados para a entrevista estruturada com crianças vítimas de violência. O procedimento consistiu em verificar nas bases de dados eletrônicas Bireme, INDEXPSI, SciELO, PePSIC, PubMed, Web of Science e PsycINFO, utilizando-se como descritores as palavras NICHD e sexual abuse, para artigos publicados entre os anos de 2000 e 2013. Foram consultados também acervos de livros em universidades. Nas bases de dados relacionadas, foram encontrados 73 artigos, dos quais 47 foram excluídos tendo como critério de exclusão artigos relacionados a outras temáticas de violência que não a sexual, e artigos repetidos, resultando em 26 artigos consultados. Nos acervos de Universidades foram consultados quatro livros nacionais, quatro livros internacionais e uma dissertação de mestrado, resultando em um total de 35 publicações analisadas. O protocolo apresenta-se como um instrumento estruturado, transmitindo informações de qualidade superior àquelas obtidas por entrevistas com menos questões abertas. Como resultado são produzidas respostas mais precisas, com relatos mais detalhados e ricos e com maior número de revelações.

Palavras-chave: Abuso sexual infantil, oitiva de crianças, Protocolo NICHD.

Endereço para correspondência: Centro de Educação e Ciências Humanas, Programa de Pós-Graduação em Psicologia, Laboratório de Análise e Prevenção da Violência, Universidade Federal de São Carlos, Rod. Washington Luis, km 235, São Carlos, SP, Brasil 13565-905. E-mail: laprev_ufscar@yahoo.com.br Apoio financeiro da Fundação de Amparo à Pesquisa do Estado de São Paulo (FAPESP). Processo $n^{\circ}$ 2013/06690-9.

Apoiado pela Fundação da Ciência e Tecnologia (FCT; PTDC/MHC-PAP/4295/ 2012) e pelo Programa Operacional Fatores de Competitividade (COMPETE; CCOMP -01-0124-FEDER-029554). 


\title{
Investigation of Suspected Child Sexual Abuse: The NICHD Protocol
}

\begin{abstract}
This article consists of a systematic literature review on best practices regarding investigative interviews of child sexual violence, with emphasis on the NICHD Protocol (National Institute of Child Health and Human Development), recognized by the international specialized literature as the most appropriate structured interview for victimized children. The method consisted in checking the electronic databases Bireme, INDEXPSI, SciELO, PePSIC, PubMed, Web of Science and PsycINFO, using the keywords NICHD and sexual abuse for papers published from 2000 to 2013. Books pertaining to University libraries in Brazil were also consulted. In the database, 73 articles were found, 47 were excluded as they related to other themes other than sexual violence or were repeated, resulting in 26 studies. Four Brazilian books, four North-American ones, and one M.A. thesis were consulted in the University libraries, resulting in a total of 35 publications. The protocol is presented as a structured instrument, which gathers superior information in comparison to interviews with fewer open-ended questions. As a result, more accurate answers are produced, with more detailed and richer accounts providing a higher frequency of disclosures.
\end{abstract}

Keywords: Child sexual abuse, children testimony, NICHD Protocol.

\section{Investigación de Sospecha de Abuso Sexual Infantojuvenil: EI Protocolo NICHD}

\section{Resumen}

Este artículo consiste en una revisión sistemática de la literatura sobre la escucha y estrategias adecuadas para la investigación de la violencia sexual infantil, con especial énfasis en el Protocolo NICHD (Instituto Nacional de Salud Infantil y Desarrollo Humano), el cual es reconocido y validado como la herramienta más apropiada que entrevistas estructuradas para niños y niñas víctimas de violencia. El procedimiento consistió en la verificación de las siguientes bases de datos electrónicas INDEXPSI Bireme, SciELO, PePSIC, PubMed, Web of Science y PsycINFO, usando como descriptores abuso NICHD y sexual, de los artículos publicados entre 2000 y 2013. También se fueron consultados colecciones de libros en las universidades. En las bases de datos, se encontraron 73 artículos, de los cuales 47 fueron excluidos por estar relacionados con otros temas de la violencia sin ser la sexual, ser repetidos, dando lugar a 26 artículos encontrados. En las colecciones de la universidad se consultó cuatro libros nacionales, cuatro libros e internacionales y una tesis, lo que resulta en 35 publicaciones analizadas. El protocolo se presenta como un instrumento estructurado y transmite información superior a los obtenidos mediante entrevistas a las preguntas menos abiertas. Como resultado son producidas respuestas por lo tanto más precisas, obteniendo relatos más detalladas y con más riqueza en las revelaciones.

Palabras clave: Abuso sexual en jóvenes, escucha de niños y niñas, Protocolo NICHD.

É evidente a necessidade de proteção da criança ou adolescente em situação de violência. A Constituição Federal (Constituição da República Federativa do Brasil, 1988/1997), no artigo 227 , dispõe que a criança deve ser colocada a salvo de qualquer forma de violência, sendo punidos severamente o abuso e a exploração sexual da criança e do adolescente. Para se efetivar a proteção, foi instituída a Lei de número 8.069, denominada de Estatuto da Criança e do Adolescente - ECA (1990), que, no seu artigo 18 , dispõe que crianças e adolescentes devem 
estar protegidos de qualquer tratamento desumano, violento, aterrorizante, vexatório e constrangedor, devendo sua dignidade ser garantida. A notificação da violência é obrigatória (artigo 245) e cabe aos Conselhos Tutelares adotarem as medidas protetivas cabíveis e incluir a criança na Rede de Proteção. Visto que o país dispõe de leis para a proteção dos direitos da criança, espera-se que a lei seja cumprida e para tal é preciso que a ocorrência do abuso sexual (AS) seja comprovada judicialmente e que o melhor interesse da criança seja atendido.

A fim de alcançar tal objetivo existem alguns desafios a superar, já que a literatura especializada aponta que o AS não deixa marcas físicas ou sinais de doenças sexualmente transmissíveis em aproximadamente $80 \%$ dos casos (Salvagni \& Wagner, 2006).

É possível verificar por meio de diversos estudos como os de Brown e Lamb (2009), Cunningham (2009), Dobke (2001), Herman (2009) e Oliveira (2010) que a exigência exclusiva de provas médicas materiais que comprovem a agressão inviabiliza a realização de um trabalho efetivo voltado para a proteção da vítima.

Dessa forma, para que os procedimentos jurídicos gerem a responsabilização do agressor e proteção da vítima, a Psicologia poderá contribuir com práticas que auxiliem o andamento do caso (Pelisoli, Gava, \& Dell'Aglio, 2011), considerando que a ausência de materialidade do crime leva a uma centralidade do depoimento da criança nas diferentes fases do processo.

Williams (2009) aponta como exemplo dessa realidade o estudo de Caldeira, realizado em 2005 na cidade de São Carlos, SP, o qual corrobora os autores citados ao apontar que, dentre 37 casos denunciados de AS no ano de 2003, $67,5 \%$ não foram encaminhados ao Fórum por não apresentarem provas materiais. Dentre os casos citados, somente 12 foram encaminhados, porém oito desses foram arquivados pelo mesmo motivo. Finalmente, entre os três casos julgados, dois agressores foram absolvidos também por falta de provas e apenas um desses foi condenado (ponderando que este confessou a agressão e teve como pena o pagamento de uma multa de pequeno valor monetário).
Ofensores sexuais de crianças dificilmente confessam esse crime, a menos que possam obter alguma vantagem como a redução da pena, após negociação com o Ministério Público. Devido à falta de motivação do suposto agressor em confessar o crime, ou a presença pouco frequente de testemunhas oculares, apenas com o depoimento da criança podem ser coletadas informações que permitam contextualizar evidências, identificando o agressor, os espaços, o tempo, as práticas e consequências do alegado abuso sexual, mesmo quando existem evidências físicas de sua ocorrência.

Além das dificuldades encontradas em relação às provas nessa modalidade de violência, os casos não solucionados no Brasil se dão devido à indisponibilidade de um procedimento padrão que seja validado e preciso para a obtenção do relato da criança. Consequentemente, a criança vem a ser entrevistada por profissionais de diversas áreas de atuação, os quais não são treinados para tal, utilizando práticas que a inibem, traumatizam e/ou induzem-na a relatar fatos que não ocorreram. Tais entrevistas são realizadas em contextos diferentes, como Conselhos Tutelares, delegacias, perícias, Vara da Infância e da Juventude, Vara Criminal e Vara de Família (Santos \& Gonçalves, 2008), acontecendo de maneira repetitiva e revitimizante, com o agravante de tornar os danos primários mais traumáticos.

De acordo com Goodman e Quas (2008), a precisão da memória infantil de eventos está relacionada com a forma com que a entrevista ocorre e não com a quantidade de entrevistas realizadas. É importante destacar que a intensidade dos danos secundários para a vítima de abuso sexual é proporcional à sua fragilidade e vulnerabilidade, assim a criança tem um papel fundamental nos processos e precisa ser assegurada por um atendimento profissional (Peixoto, Ribeiro, \& Lamb, 2011).

No Brasil, a oitiva das vítimas em audiências criminais deve ser realizada na modalidade de Depoimento Especial, segundo recomendação do Conselho Nacional de Justiça (resolução 33/2010). Entretanto, em alguns locais ainda tem sido realizada da mesma maneira que ocorre em processos de violência sexual nos quais 
a vítima adulta, destacando o despreparo dos operadores do Direito para lidar com o assunto (Dobke, 2001). De cordo com Cezar (2007), o Depoimento Especial (denominado incialmente de Depoimento sem Dano) é a oitiva de crianças em processos criminais, sendo realizada em ambiente separado da sala de audiências, onde a criança interage com psicólogo ou assistente social que fará as perguntas formuladas pelo Juiz, pelo Promotor e pelo Defensor do réu. A interação é transmitida em tempo real à sala de audiências por meio de circuito fechado de imagem e som, o que permite o direito à ampla defesa do réu e ao contraditório (Constituição Federal, 1988/1997). Tal método tem o objetivo de diminuir a revitimização e melhorar a qualidade da produção da prova. Inquirições realizadas na presença do réu representam um dos impedimentos para que a criança expresse os fatos devido ao medo, sendo essa uma das causas da não punição do agressor.

Além dos profissionais envolvidos com oitivas de crianças em audiências criminais, psicólogos da Vara da Família, bem como da Vara da Infância e da Juventude, também podem se deparar com a necessidade de realizar a escuta de crianças relacionada ao abuso sexual supostamente sofrido, pois, não raro, em casos de AS intrafamiliar pode haver disputas pela guarda da criança e a necessidade de se avaliar o risco em permitir a aproximação da criança com um pai supostamente agressor (Volnovich, 2005; Wolfe, 1998).

Essa possibilidade de reaproximação impõe o desenvolvimento de boas práticas de avaliação do caso, entre elas a entrevista com a criança para revelação de AS (Padilha \& Antunes, 2009).

Adicionalmente, de acordo com Pelisoli et al. (2011), no contexto jurídico, fatores como a utilização de heurísticas e de crenças prévias podem influenciar a tomada de decisão por parte do psicólogo na sua avaliação. Logo, a utilização de metodologia sistematizada e baseada em evidências poderá controlar a ocorrência de processo de decisão a priori sobre este tipo de casos.

Nesta perspectiva, e numa problemática na qual a palavra da criança assume um papel central, faz-se necessária a adoção de protocolos de entrevista forense que facilitem a coleta do relato da criança, minimizem a possibilidade de induções de falsos relatos e, ao mesmo tempo, protejam a criança contra revitimizações, por evitar que tenha que ser ouvida diversas vezes em função da qualidade questionável de algumas entrevistas.

Importa aqui definir aquilo que se entende por entrevista forense. Este termo designa uma entrevista que tem como objetivo a coleta de informações que são relevantes para a compreensão de um determinado acontecimento, na maior parte das vezes, com implicações para uma investigação criminal ou numa investigação que visa a proteção da criança. Esta entrevista é centrada nos fatos, nos atores, nos locais, no tempo, nas ações e suas consequências. Deve-se distinguir este tipo de entrevista de outras que são mais habituais ao psicólogo, como a entrevista clínica, centrada nas informações que retratam a trajetória desenvolvimental da criança, ou uma entrevista terapêutica, com o objetivo de ajudar a criança em seus problemas emocionais e comportamentais (Peixoto, Ribeiro, \& Magalhães, 2013). O uso da entrevista forense pelo psicólogo que trabalha no âmbito da justiça é fundamental no sentido de uma melhor e mais fiável compreensão do que aconteceu à criança, definindo toda a intervenção ulterior do psicólogo e dos profissionais que a atenderão, quer num papel de avaliação psicológica forense, quer num papel clínico e psicoterapêutico.

\section{Protocolos de Entrevista Estruturada para Investigação de Violência Sexual Infantil}

Motivados em parte pela ampla publicidade sobre os casos de abuso sexual em crianças e adolescentes no exterior sem solução e sem a devida materialização das provas, diversos autores (Harris, Goodman, Augusti, Chae, \& Alley, 2009; Klemfuss \& Ceci, 2009; La Rooy, Lamb, \& Pipe, 2009 e Lamb, Orbach, Hershkowitz, Esplin, \& Horowitz, 2007), têm estudado a capacidade de crianças em fornecer informa- 
ções mais precisas sobre suas experiências passadas e sobre a sugestionabilidade a que estão suscetíveis.

Conforme Klemfuss e Ceci (2009), cabe ao profissional ponderar a respeito das práticas utilizadas em entrevistas com crianças vítimas de AS, a fim de realizar uma escuta livre de sugestões e que seja realizada utilizando o mínimo de questões fechadas possível.

Segundo Goodman, Ogle, Troxel, Lawler e Cordon (2008), de forma geral os protocolos de entrevista forense devem incluir os seguintes procedimentos: estabelecimento de confiança, avaliação de desenvolvimento, avaliação sobre a capacidade de a criança distinguir entre verdade e mentira, informações sobre a entrevista, possibilidade de dizer "não sei", perguntas abertas/neutras e encerramento. A maioria dos protocolos de entrevista forense com crianças propõe que seja realizada apenas uma entrevista.

Dentre os diversos tipos de protocolos de entrevista, deve-se destacar: o Achieving Best Evidence in Criminal Proceedings - Guidance on Interviewing Victims and witnesses, and Using Special Measures (Home Office, 2011); sucessor do Memorandum of Good Practice (Home Office, 1992); a Entrevista Cognitiva (EC; Fisher \& Geiselman, 1992); a Entrevista Passo-a-Passo (Yuille, Hunter, Joffe, \& Zaparniuk, 1993); o Protocolo de entrevista forense da Corner House - RATAC (Finding Words; Anderson et al., 2010); e o Protocolo NICHD (National Institute of Child Health and Human Development; Lamb, Hershkovitz, Orbach, \& Esplin, 2008). Contudo, na literatura sobre entrevista forense de crianças, a EC e o Protocolo NICHD destacam-se pela existência de um maior número de estudos empíricos realizados.

A pedido da polícia norte-americana e dos operadores do direito, Ronald Fisher e Edward Geiselman, desenvolveram em 1984 uma técnica conhecida como Entrevista Cognitiva (EC; Feix \& Pergher, 2010), originalmente elaborada para entrevistar adultos. Esta técnica foi revisada em 1992 (Fisher \& Geiselman, 1992). A EC tem como propósito obter depoimentos com mais de- talhes e precisão, sendo as entrevistas gravadas para evitar falhas da memória do entrevistador e interpretações equivocadas.

A EC é composta de cinco etapas (Rapport, Recriação do Contexto Original, Narrativa Livre, Questionamento e Fechamento) que procuram unir conhecimentos da Psicologia Social com a Psicologia Cognitiva para uma maior precisão dos relatos (Feix \& Pergher, 2010). Estudos demonstram que a EC é uma técnica útil em contexto forense (Memon, Meissner, \& Fraser, 2010), porém deve ser utilizada com cuidado, pois pode ocorrer certa confabulação em crianças mais novas diminuindo a precisão dos resultados obtidos (Cronch, Viljoen, \& Hansen, 2006).

O protocolo NICHD foi desenvolvido após a constatação de que os profissionais responsáveis por entrevistas com crianças vítimas de AS tinham significativa dificuldade em aderir às recomendações de boas práticas em entrevistas investigativas, como por exemplo, a necessidade de se reduzir o número de questões sugestivas, fechadas e de múltipla escolha, aumentando o número de questões abertas. Os profissionais que seguiam tais recomendações obtiveram resultados mais precisos (Lamb et al., 2008). O protocolo NICHD se assemelha em muitos aspectos com a Entrevista Cognitiva (Fisher \& Geiselman, 1992), porém, a última carece de uma especificação detalhada de como o entrevistador deve formular as questões. Em contraste, o protocolo NICHD apresenta questões específicas para o treino de memória episódica, o que aumenta a efetividade da entrevista (Lamb et al., 2008).

Frente à necessidade de definição de boas práticas para a entrevista forense com crianças vítimas de abuso sexual no Brasil, revela-se importante demonstrar que o Protocolo NICHD tem sido objeto de pesquisa há vários anos, em diversos países. O objetivo do presente estudo é descrever brevemente o Protocolo NICHD, rever a literatura sobre pesquisas realizadas com o mesmo e apontar as possibilidades de sua utilização à realidade brasileira. 


\section{Método}

Para a elaboração do presente trabalho, foram pesquisados artigos da literatura especializada em abuso sexual nas bases de dados eletrônicas Bireme, INDEXPSI, SciELO, PePSIC, PubMed, Web of Science e PsycINFO, utilizando-se como descritores as palavras NICHD e sexual abuse (AS), para artigos publicados entre os anos de 2000 e 2013. Foram consultados também acervos de livros nas Universidades dos autores. Nas bases de dados relacionadas, foram encontrados 73 artigos, dos quais 47 foram excluídos tendo como critério de exclusão artigos relacionados a outras temáticas de violência que não a sexual e artigos repetidos, resultando em 26 artigos consultados. Nos acervos de Universidades foram consultados quatro livros nacionais, quatro livros internacionais e uma dissertação de mestrado, resultando em um total de 35 publicações analisadas.

\section{Resultados}

De acordo com a revisão bibliográfica, foi possível verificar que, no ano de 1996, pesquisadores do National Institute of Child Health and Human Development (Instituto Nacional da Saúde Infantil e Desenvolvimento Humano - NICHD) deram início a estudos realizados nos Estados Unidos para o desenvolvimento de entrevistas investigativas do abuso sexual infantil contando inicialmente com a participação de Michael E. Lamb, Yael Orbach e Kathleen Sternberg, bem como Irit Hershkowitz, pesquisadora da Universidade de Haifa em Israel; Phillip Esplin, psicólogo forense e Dvora Horowitz, funcionária do governo Israelense.

O protocolo de entrevista recebeu a mesma denominação do Instituto, ou seja, Protocolo de
Entrevista Investigativa NICHD e está alicerçado na operacionalização das recomendações de pesquisadores para auxiliar nas investigações forenses ao conduzir as entrevistas com as crianças vítimas de AS.

$\mathrm{O}$ protocolo tem sido replicado e validado nos estudos de Lamb et al. (2008; Lamb et al., 2007) em países como Estados Unidos da América, Suécia, Finlândia, Noruega, Canadá, Reino Unido e Israel. Em estudos de revisão realizados por Lamb et al. (2008; Lamb et al., 2007), foi observado em mais de 40.000 entrevistas que o uso do protocolo melhorou drasticamente a qualidade da entrevista de investigação em diversos países. Os estudos realizados com o protocolo no campo forense ajudaram a gerar um consenso entre os profissionais envolvidos a respeito das competências e limitações das crianças. Assim há evidências claras de que o protocolo NICHD pode ser usado de forma produtiva em uma variedade de contextos culturais (Aldridge et al., 2004; Hershkowitz, Fisher, Lamb, \& Horowitz, 2007; Hershkowitz, Horowitz, \& Lamb, 2005; Hershkowitz, Horowitz, Lamb, Orbach, \& Sternberg, 2004; Hershkowitz, Lanes, \& Lamb, 2007; Hershkowitz, Orbach, Lamb, Sternberg, \& Horowitz, 2006; Lamb \& Garretson, 2003; Lamb et al., 2007; Lamb et al., 2003; Malloy, Brubacher, \& Lamb, 2011 e Peixoto, Ribeiro, \& Alberto, 2013).

Dentre os 20 estudos encontrados na presente revisão envolvendo especificamente $o$ protocolo NICHD, a maioria configurou-se como estudo empírico, totalizando 15 estudos. Adicionalmente, foram encontrados quatro estudos de revisão de literatura e dois estudos teóricos. A Tabela 1 resume a descrição dos estudos pesquisados, seus objetivos, método e principais resultados. 
Tabela 1

Comparação entre os Estudos Encontrados Envolvendo o Protocolo NICHD

\begin{tabular}{llll}
\hline Estudo Característica & Objetivo & Método & Principais Resultados
\end{tabular}

\begin{tabular}{lll}
\hline Lamb et al., & Estudo & Verificar a \\
1996 & Empírico & influência do \\
& & estilo de entrevista \\
& investigativa para \\
& obtenção de relatos \\
& sobre AS
\end{tabular}

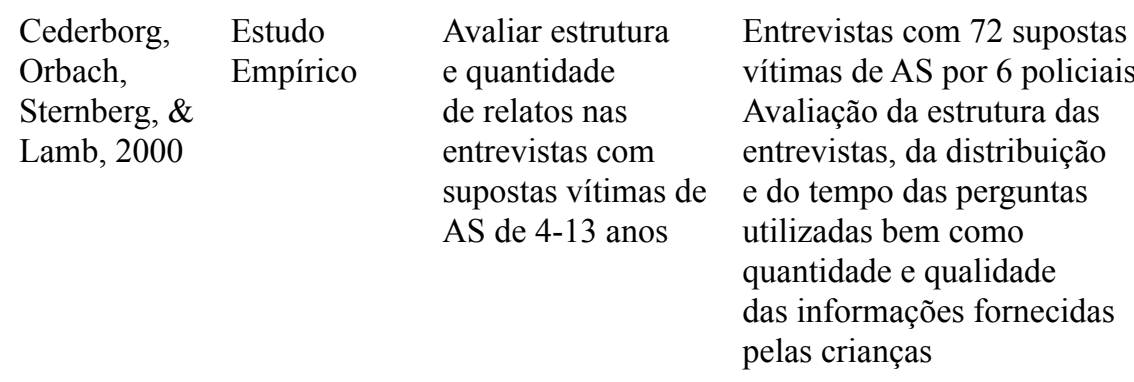

Orbach et al., Estudo Avaliar eficácia $2000 \quad$ Empírico

$\begin{array}{ll}\text { Lamb \& } & \text { Estudo } \\ \text { Garretson, } & \text { Empírico } \\ 2003 & \end{array}$
entrevistaram 672 supostas
Análise psicolinguística detalhada de 22 entrevistas realizadas com crianças de 5-11 anos categorizadas de acordo com a extensão e riqueza do relato considerando os diferentes tipos de perguntas utilizadas as crianças mais velhas

Verificar a
influência
que o sexo do
entrevistador
e o sexo da
criança exercem
no processo de
investigação de AS

Investigadores forenses vítimas nas idades entre 4-14 anos. Trezentos e cinquenta entrevistas foram realizadas com o protocolo e $322 \mathrm{sem}$ o mesmo fechadas ou sugestivas,
Entrevistadores utilizaram principalmente perguntas fechadas e sugestivas (53\%). Seis \% das perguntas feitas pelos entrevistadores eram abertas eliciando apenas $8 \%$ das informações obtidas.

Necessidade de práticas de entrevista que melhorem a qualidade das informações fornecidas

As entrevistas com o protocolo continham mais perguntas abertas do que as entrevistas sem o mesmo. Mais detalhes foram obtidos utilizando perguntas abertas, com ou sem o protocolo. Em ambas as condições forneceram mais detalhes do que as mais novas

Perguntas abertas renderam mais extensas e detalhadas do que as perguntas independentemente da idade

As análises das entrevistas mostraram efeitos

significativos

de gênero, tanto no comportamento dos entrevistadores como na quantidade de informações relatadas pelas crianças. As entrevistadoras fizeram mais questões abertas e sugestivas para meninos do que para meninas. Entrevistadores do sexo masculino entrevistaram meninos e meninas da mesma forma e as respostas das crianças variaram em função do sexo e idade, sexo do entrevistador e tipo de pergunta utilizada. Influência do sexo do entrevistador foi atenuada em entrevistas guiadas pelo protocolo 


$\begin{array}{lll}\text { Lamb et al., } & \text { Estudo } & \text { Determinar } \\ & \text { Empírico } & \text { se crianças } \\ & \text { que prestaram } \\ & \text { depoimento no } \\ & \text { fórum sobre casos } \\ & \text { de AS eram mais } \\ & \text { ou menos capazes } \\ & \text { de informar sobre } \\ & \text { os incidentes que as } \\ & \text { supostas vítimas }\end{array}$

\begin{tabular}{|c|c|c|c|}
\hline $\begin{array}{l}\text { Aldridge } \\
\text { et al., } 2004\end{array}$ & $\begin{array}{l}\text { Estudo } \\
\text { Empírico }\end{array}$ & $\begin{array}{l}\text { Verificar a precisão } \\
\text { das entrevistas } \\
\text { com o protocolo } \\
\text { quando associadas a } \\
\text { desenhos da figura } \\
\text { humana }\end{array}$ & $\begin{array}{l}\text { Noventa supostas vítimas } \\
\text { de AS entre } 4-13 \text { anos } \\
\text { entrevistadas por policiais. } \\
\text { Na sequência foram } \\
\text { apresentados desenhos da } \\
\text { figura humana e realizadas } \\
\text { algumas perguntas }\end{array}$ \\
\hline
\end{tabular}

Hershkowitz Revisão de Investigar et al., 2005 Literatura características de casos de suspeita de AS associados à investigações formais

\author{
Hershkowitz Estudo \\ et al., 2006 Empírico
}

\begin{abstract}
Explorar diferenças estruturais entre entrevistas forenses em que as crianças relataram abuso e nas entrevistas que não relataram
\end{abstract}

Vinte e seis supostas vítimas de AS e 26 crianças que testemunharam situações de AS foram entrevistadas por investigadores que utilizaram o protocolo. As crianças dos 2 grupos foram pareadas em relação à sua idade, à relação com o suposto autor e com a gravidade das supostas agressões

Noventa supostas vítimas de AS entre 4-13 anos $\mathrm{Na}$ sequência foram figura humana e realizadas gumas perguntas

Banco de dados de todos os casos de suspeita de abuso físico e sexual investigados em Israel entre os anos 1998-2002 envolvendo 26.446 crianças
Testemunhas e vítimas relataram quantidades similares de informações sobre o abuso, comprovando que as crianças podem ser testemunhas para relatar $\mathrm{s}$ eventos abusivos

Desenhos associados às questões provocaram média de 86 novos detalhes forenses relevantes. Crianças de 4-7 anos forneceram média de 95 detalhes adicionais após o desenho ter sido introduzido. Como as informações relatadas com o desenho podem ter sido menos precisas (memória de reconhecimento predominante), os desenhos só devem ser introduzidos no final das entrevistas investigativas

Sessenta e cinco \% das crianças fizeram alegações de alguma modalidade de abuso, porém as taxas de relato foram maiores no caso de AS (71\%) e de abuso físico (61\%). Em qualquer idade as crianças eram menos propensas a relatar sobre o abuso quando pai era o autor. O número de revelações foi maior por parte das crianças maiores

Comparadas 50 entrevistas forenses de supostas vítimas de abuso que não fizeram

Constatado que nas entrevistas em que não houve revelação, revelação durante a entrevista os entrevistadores utilizaram com 50 vítimas que relataram menos perguntas abertas AS ou abuso físico. A partir de gravações em áudio, perguntas do entrevistador e respostas das crianças foram examinadas e menos comentários de apoio do que ao entrevistar crianças que fizeram alegações de abuso 


\author{
Hershkowitz, Estudo \\ Fisher, et al., Empírico \\ 2007
}

\author{
Hershkowitz, Estudo \\ Lanes, et al., Empírico \\ 2007
}

Lamb et al., 2007

Revisão de
literatura

Cyr \& Lamb, Estudo 2009
Investigar se a credibilidade dos relatos das crianças é maior quando são utilizadas entrevistas investigativas com o protocolo

Examinar características dos relatos das crianças feitos para os pais em casos de AS extrafamiliar
Quarenta e dois policiais avaliaram a credibilidade dos relatos de AS utilizando como instrumento a Análise de Validade da Declaração (SVA) e o Critério Baseado em Análise de Conteúdo (CBCA) para comparar 678 entrevistas realizadas com e sem o protocolo

Trinta supostas vítimas de AS e seus pais, foram entrevistadas por 6 entrevistadores utilizando o protocolo. Aos pais foi solicitado que descrevessem em detalhes o que tinha acontecido As principais características relatadas pelas crianças e pelos pais foram codificadas por dois avaliadores independentes

Apresentar resultados de pesquisas com o protocolo, relacionadas a memória infantil, habilidades comunicativas e conhecimento como possíveis diretrizes para melhorar qualidade de entrevistas forenses

Avaliar eficácia de treinamento para o uso do protocolo para policiais e profissionais da saúde mental
Comparação de 83 entrevistas realizadas antes e depois da capacitação

A maioria das entrevistas conduzidas sem o protocolo foram classificadas como impassíveis de decisão. Relatos de entrevistas com o protocolo foram mais precisamente classificados como verdadeiros

Cinquenta e três \% das crianças levaram entre 1 semana a 2 anos para fazer o relato aos pais. Menos da metade relatou primeiramente aos pais $\mathrm{e}$ mais de $40 \%$ não relatou espontaneamente. Cinquenta $\%$ das crianças relataram sentir medo ou vergonha dos pais e seus pais tenderam culpá-las ou expressaram raiva. Característica do relato variou de acordo com idade das crianças, gravidade e frequência do abuso, expectativas sobre as reações parentais, identidade do suspeito e estratégias usadas para manter o abuso em segredo

Estudos têm mostrado que a qualidade das entrevistas aumenta quando os entrevistadores utilizam o protocolo de forma fidedigna. Nenhuma outra técnica tem sido comprovada de maneira igualmente eficaz

As entrevistas realizadas após a capacitação foram mais eficazes, visto que o número de detalhes relatados teve aumento significativo 
Lyon, Lamb, Estudo \& Myers, Teórico 2009

$\begin{array}{ll}\text { Malloy et al., } & \text { Estudo } \\ 2011 & \text { Empírico }\end{array}$

Toth, $2011 \quad$ Estudo

Teórico

Comparação dos Protocolos NICHD e RATAC

Investigar quais são as consequências esperadas pelas crianças em relação ao abuso

Abordar críticas ao protocolo no estudo de Vieth (2008)
Katz \& Estudo Hershkowitz, Empírico 2012

Olafson, 2012
Revisão de Literatura
Explorar frequência e efeitos das perguntas múltiplas no relato de crianças vítimas de AS entrevistadas com o protocolo

Comentar sobre desequilíbrios relacionados à especificidade e sensibilidade nas investigações forenses
Boletim informativo da ISPCAN (International Society for the Prevention of Child Abuse and Neglect)
Principais críticas abordadas: Foram refutadas todas o protocolo não é o único as críticas, com base em que oferece suporte contínuo; argumentos dos autores que não é validado pelo sistema mostravam a inconsistência jurídico americano; as das mesmas

instruções que o protocolo

contestadas em tribunal, entre outras

Duzentos e quatro crianças, suspeitas vítimas de AS infantil, entre 5-13 anos de idade foram entrevistadas

As consequências esperadas foram mencionadas em quase metade das entrevistas e na maioria delas, espontaneamente. As crianças mais velhas foram mais propensas a relatar do que as mais jovens. As consequências mais comuns relatadas foram prejuízos físicos e emoções negativas para a própria criança e a prisão ou outra punição legal para o suspeito

Afirma que o Protocolo NICHD é mais pesquisado e oferece melhor suporte para conduzir entrevistas forenses com crianças em casos de AS. O grande número de questões abertas, ao invés de somente questões sugestivas ou fechadas a respeito do evento, possibilita que o protocolo transmita informações de qualidade superior

Uma média de 5,58 perguntas múltiplas foi identificada nas entrevistas, apresentando efeitos prejudiciais para a extensão e para a riqueza dos relatos. avaliadores identificaram perguntas simples e múltiplas Crianças de todas as idades para analisar respostas das crianças falharam em sinalizar falta de compreensão sobre perguntas múltiplas e $24 \%$ de suas respostas eram incompreensíveis.

Adaptações ou estratégias precisam ser desenvolvidas e testadas para os casos em que não há revelação; quando essa é parcial; para casos de crianças muito pequenas; com deficiência intelectual e para crianças cuja suspeita de AS envolve disputa de guarda 


$\begin{array}{lll}\text { Phillips, } & \text { Estudo } & \text { Examinar } \\ \text { Oxburgh, } & \text { Empírico } & \text { influências que o } \\ \text { Gavin, \& } & & \text { tipo de questões } \\ \text { Myklebust, } & & \text { utilizadas nas } \\ 2012 & & \text { entrevistas } \\ & \text { investigativas } \\ & & \text { exercem nos relatos } \\ & & \text { de informações } \\ & \text { relevantes }\end{array}$

Lamb, Estudo Hershkowitz, Empírico \& Lyon, 2013

Pipe, Orbach, Estudo Lamb, Empírico Abbott, \& Stewart, 2013

\author{
Vinte e duas crianças de \\ diferentes faixas etárias \\ foram entrevistadas por 21 \\ policiais ingleses
}

Análise da fase de construção do vínculo por meio de quase 200 entrevistas realizadas com crianças que relataram abuso

proporciona mais apoio do que o protocolo original

\author{
Um número maior de \\ informações relevantes \\ foi produzido ao utilizar \\ questões abertas em vez \\ de perguntas fechadas, \\ sugestivas ou perguntas \\ múltiplas. Crianças mais \\ velhas relataram mais \\ informações relevantes
}

\author{
Entrevistas com protocolo \\ revisado favoreceram o \\ vínculo em comparação ao \\ protocolo original, além de \\ ter sido constatado menos \\ sinais de resistência pelas \\ crianças e um aumento na \\ quantidade de revelações \\ $(18,8 \%)$ \\ Revelações eram mais \\ entrevistadores foram \\ capacitados. Houve aumento \\ relevante no número de \\ sentenças condenatórias \\ quando o protocolo foi \\ utilizado, apontando para \\ importantes implicações \\ nas políticas relativas à \\ investigação de crimes de AS
}

Comparação das entrevistas realizadas por 16 policiais em prováveis quando os casos de suspeita de AS com 760 crianças de 3-14 anos de idade, entre os anos de 1994 à 1997, antes do treinamento do protocolo $(n=350)$ e após o treinamento $(n=410)$ entre os anos de 1997-2000
Nota-se na Tabela 1 semelhanças nos resultados de estudos realizados por diversos autores (Aldridge et al., 2004; Cyr \& Lamb, 2009; Hershkowitz, Fisher, et al., 2007; Hershkowitz et al., 2005; Hershkowitz et al., 2006; Lamb et al., 1996; Lamb et al., 2007; Orbach et al., 2000; Peixoto, Ribeiro, \& Alberto, 2013; Phillips et al., 2012 e Toth, 2011), os quais afirmam ser possível quantificar um maior número de relatos e de detalhes forenses relevantes em entrevistas com o protocolo. Foram também apontadas limitações do protocolo NICHD (Lamb et al., 2013 e Olafson, 2012), apontando que o mesmo pode não obter bons resultados com crianças resistentes em fazer a revelação ou fazê-la parcialmente; crianças muito pequenas; com deficiência intelectual e crianças envolvidas em disputa de guarda. É importante ressaltar que a publicação de pesquisas relacionadas ao protocolo NICHD é ainda inexistente no Brasil, portanto, a tabela apresenta apenas estudos internacionais.
Em sua estrutura de investigação, o protocolo NICHD abarca conhecimentos sólidos sobre o desenvolvimento infantil, de acordo com características individuais da vítima e observa alguns aspectos na aplicação, tais como: a linguagem, verificando em que estágio do desenvolvimento a criança se encontra; a memória, caracterizada por estágios e influenciada pelo grau de estresse gerado pela violência sexual infantil; a pressão exercida por membros familiares e pelo próprio agressor; a influência da fase do desenvolvimento em que a criança se encontra na maneira como se comporta; e o impacto emocional que pode gerar distúrbios de comportamento. Outro aspecto importante na concepção e operacionalização do protocolo é a utilização de processos de evocação livre como principal estratégia na obtenção de informação (Orbach \& Pipe, 2011). Assim, a utilização de questões abertas tem como propósito ser a principal estratégia de estimulação da evocação livre por parte da criança 
de acontecimentos da sua vida. Para além disso, esta estratégia é também a melhor forma de estimular a construção de narrativas (Roberts, Brubacher, Powell, \& Price, 2011), e providencia uma melhor qualidade e quantidade de informação (Lamb et al., 2008). A utilização de questões abertas surge como a melhor alternativa à utilização de processos que utilizam uma memória de reconhecimento, mais concretamente, a colocação de questões de múltipla escolha ou sugestivas. Essas últimas têm sido apontadas como estratégias que aumentam a quantidade de erros apresentados pela criança (Ceci \& Bruck, 1995), e limitadoras da quantidade de informação que a criança poderá fornecer (Orbach \& Pipe, 2011). De fato, a utilização de questões de múltipla escolha ou de questões sugestivas facilita a aquiescência da criança (sobretudo perante adultos em posição de autoridade; Ceci \& Bruck, 1995); provoca um falso reconhecimento de detalhes (por vezes centrais, como a identidade do ofensor); ou leva a criança a fornecer informações que não decorrem da sua memória episódica, mas sim de uma síntese dos acontecimentos vivenciados (Brainerd \& Reyna, 2005).

O protocolo NICHD tem como premissa realizar a investigação da suspeita de violência sexual infantil de forma estruturada abarcando todas as fases necessárias para uma entrevista investigativa. Lamb et al. (2008), dividiram o protocolo em duas partes, a parte pré-substantiva e a parte substantiva. A parte pré-substantiva conta com algumas etapas:

1. Etapa introdutória, na qual o entrevistador se apresenta à criança e esclarece a tarefa que será realizada, ou seja, a necessidade de falar a verdade e descrever eventos em detalhes. Ainda nessa fase, o entrevistador explica as regras básicas e as expectativas, como por exemplo, de que a criança deve dizer que não se lembra de algum evento, que não sabe a resposta, que não entendeu a pergunta ou que corrija o entrevistador quando for apropriado;

2. Construção do vínculo, que compreende duas subdivisões: (a) criar um ambiente descontraído e de apoio para estabelecer o rapport entre a criança e o entrevistador; e (b) solicitar que a criança descreva experiências recentes e eventos neutros em detalhes. Nessa parte da entrevista, o objetivo da sessão é que a criança se familiarize com as questões abertas e com as técnicas que serão utilizadas na próxima etapa, definida como substantiva e descrita posteriormente.

Ainda na fase pré-substantiva, uma série de instruções são utilizadas de forma não sugestiva para identificar eventos alvo que estão sob investigação. As questões são as mais abertas possíveis e o entrevistador só avança para perguntas mais focadas, se a criança não apresentar falhas em identificar o evento alvo.

$\mathrm{Na}$ eventualidade de existirem evidências claras sobre os fatos que estão sob investigação criminal (testemunhas oculares, indicadores de abuso sexual, como é o caso de uma gravidez), na fase de transição para as questões substantivas o protocolo sugere a utilização, pelo entrevistador, de parte da informação recolhida previamente com a criança, no sentido de poder desencadear uma revelação. Porém, mesmo com a utilização desta estratégia que poderá ser considerada sugestiva, o entrevistador nunca deverá fornecer informação à criança sobre o tipo de atos sofridos ou sobre a identificação do ofensor.

Assim que acontecer o primeiro relato da criança sobre o abuso sexual, o entrevistador pede à criança para indicar se o incidente ocorreu uma vez ou mais do que uma e procede para se assegurar que o incidente seja relatado utilizando a pergunta "Me conta mais sobre..." (atividade mencionada pela criança). Desse modo, o entrevistador pede que a criança fale mais sobre o evento que relatou anteriormente, utilizando as mesmas palavras da criança e fazendo referência a detalhes mencionados por ela, tomando cuidado para não contaminar as memórias referentes ao evento. Apenas após perguntas abertas exaustivas realizadas pelo entrevistador a respeito do evento relatado pela criança, o mesmo faz perguntas diretas, porém ainda tendo como parâmetro relatos da criança durante as perguntas abertas e solicitando informações específicas, como por exemplo, quando aconteceu o episódio ou sobre a aparência de determinada pessoa. 
As questões que trazem declarações sugestivas, como as que comunicam à criança a resposta esperada, são fortemente desencorajadas durante a utilização do protocolo. Concluída essa parte da entrevista, o entrevistador dá sequência, iniciando a parte substantiva com a pergunta chave "Agora que conheço você um pouco mais, queria falar sobre porque você veio aqui hoje". O entrevistador dá continuidade com as perguntas abertas para investigar os incidentes e continua a utilizar a consigna "Me conta tudo sobre isso", especificamente para as revelações ou relatos feitos pela criança, encerrando a sessão com um tópico neutro.

Para elucidar a utilização do referido protocolo, Lamb et al. (2008), assinalam o estudo realizado em Israel, no ano de 2000, no qual compararam resultados apresentados em 55 entrevistas utilizando o protocolo NICHD, com outras 50 entrevistas cujo procedimento era direcionado pelo protocolo fornecido pelo Serviço Investigativo Israelense. Ambos os procedimentos foram conduzidos pelos mesmos seis entrevistadores, os quais tinham graduação em serviço social e eram os únicos funcionários autorizados a realizar entrevistas com crianças vítimas de abuso sexual de até 14 anos de idade pelo governo de Israel. Os profissionais que realizaram as entrevistas foram capacitados para a utilização do protocolo NICHD em sessões de grupo e individuais. Os autores puderam, também, comprovar a veracidade dos relatos obtidos nas entrevistas realizadas devido ao fato de $71 \%$ dos suspeitos terem confessado o crime, relatando o episódio com os mesmos detalhes que foram relatados na alegação do evento pelas crianças durante a entrevista com o protocolo.

Outro exemplo de estudo realizado com o Protocolo NICHD foi o de Cyr e Lamb, 2009, em Quebéc, no Canadá. Foi realizada a tradução do protocolo para o idioma francês, permitindo verificar se seu uso beneficiaria o trabalho realizado por profissionais da Saúde Mental e da polícia. Policiais e assistentes sociais realizaram 83 entrevistas com crianças encaminhadas à polícia ou para os serviços de proteção à criança, utilizando o referido protocolo, pareando-as com 83 entrevistas realizadas sem o protocolo
NICHD, pelos mesmos profissionais anteriormente à capacitação. Foi constatado que o protocolo mostrou-se efetivo no sentido de obter mais relatos das crianças que sofreram abuso sexual. Tanto no estudo realizado em Israel, como no estudo canadense, os autores verificaram que as entrevistas com o protocolo continham menos perguntas fechadas e sugestivas do que nas entrevistas em que o Protocolo não foi utilizado. As crianças entrevistadas com o protocolo NICHD lembraram-se significativamente de mais detalhes de memórias com episódios neutros na fase pré-substantiva do que as crianças nas entrevistas sem o protocolo. Além do mais, foram relatados maiores detalhes pelas crianças quando utilizado o protocolo enquanto que poucos detalhes foram relatados quando utilizadas somente as questões sugestivas e fechadas.

O Protocolo NICHD claramente leva os entrevistadores nos estudos descritos por Lamb et al., 2008, a estabelecerem condições superiores para recuperação de memórias episódicas da criança de uma forma mais flexível, além de demonstrar que a técnica é eficaz por atingir a ampla faixa etária compreendida dos 4 aos 13 anos de idade. Os autores acreditam que o sucesso dos relatos se deva ao intenso treinamento, monitoramento e feedback, decorrente do tempo prolongado investido, tanto na capacitação para a prática do protocolo, como na supervisão.

Recentemente Katz e Hershkowitz (2012), realizaram um estudo a respeito dos efeitos das perguntas múltiplas estruturadas (por exemplo: "Ele tocou partes do seu corpo ou machucou alguma parte do seu corpo?"), empregadas em entrevistas realizadas com 71 crianças israelenses selecionadas considerando o critério de serem ouvidas a respeito de uma única situação de abuso por um agressor que não fosse membro da família. Foi examinado o número de palavras e de detalhes fornecidos por elas nessa modalidade de entrevista, sendo constatado que o efeito das perguntas múltiplas estruturadas em entrevistas forenses foi destrutivo e prejudicou a qualidade da entrevista com a criança. Assim, ficou patente a necessidade de se eliminar essa modalidade de entrevista por meio de constante atualização e treinamento profissional. 
Os estudos empíricos realizados com o protocolo também têm analisado a entrevista com crianças que demonstram relutância em revelar os abusos de que foram vítimas (Hershkowitz et al., 2005; Hershkowitz, Lanes, et al., 2007; Orbach, Shiloach, \& Lamb, 2007). Estes estudos indicam que vítimas relutantes evidenciam uma menor probabilidade de revelar os abusos ou relatam menos pormenores relativos a estes. Este comportamento da criança parece provocar alterações na interação com o entrevistador. Se por um lado, os entrevistadores tendem a manter o mesmo tipo de interação na fase pré-substantiva, agindo de acordo com o que é recomendado no protocolo e utilizando mais questões abertas, por outro lado, na fase substantiva, perante a resistência da criança em revelar, tendem a distanciar-se das recomendações do protocolo e a evidenciar mais questões sugestivas. A pesquisa com crianças relutantes (Lamb et al., 2013; Lamb et al., 2008) tem indicado que o um maior investimento na relação com o entrevistado parece ser o método mais eficaz para ultrapassar as resistências relativamente a falar sobre os acontecimentos abusivos. Aliás, existe já uma versão do protocolo na qual o entrevistador recorre a uma série de comentários de suporte não sugestivos. Esta versão tem alterações da dinâmica da entrevista, com um maior investimento no estabelecimento de uma atmosfera de suporte, levando a criança a sentir-se mais confortável e apoiada, facilitando, assim, a revelação (Lamb et al., 2013).

É importante considerar que os achados comprovam a efetividade do protocolo NICHD no auxílio dos entrevistadores para realizar entrevistas de acordo com as práticas recomendadas, no sentido de suscitar informações mais precisas e evitar perguntas que acarretam em informações duvidosas. Além dos autores do protocolo, outros pesquisadores (Cronch et al., 2006; Cyr, Dion, Mcduff, \& Trotier-Sylvaini, 2012; Herman, 2009; Olafson, 2012; Phillips et al., 2012; Snider \& Everson, 2011 e Toth, 2011) afirmam ser o Protocolo NICHD o mais pesquisado e aquele que oferece o melhor suporte para conduzir entrevistas forenses com crianças em casos de abuso sexual. Adicionalmente, tais autores também defendem que o grande número de questões abertas, ao invés de somente questões sugestivas ou fechadas a respeito do evento em que a agressão aconteceu, possibilita que o protocolo transmita informações de qualidade superior às que são infrequentes nas entrevistas exclusivamente sugestivas ou com opção de respostas fechadas, que maximizam a possibilidade de contaminação do relato.

Em estudo recente realizado no estado americano de Utah, Pipe et al. (2013) compararam casos de AS nos quais as entrevistas com as crianças ocorreram antes que o protocolo NICHD fosse adotado (350 casos) e depois que o protocolo foi adotado pelos entrevistadores (410 casos), para verificar se houve mudanças nos resultados de casos suspeitos no que diz respeito à instauração de processos e condenações. Os autores verificaram que, após a introdução do uso do protocolo nas entrevistas investigativas, houve uma tendência para que um número menor de casos suspeitos fosse a julgamento, sendo arquivados na fase de investigação, com a rejeição de falsos relatos. Adicionalmente, observou-se uma tendência de que a maioria dos casos que ia a julgamento resultasse em condenação do acusado, com a melhoria da qualidade da prova coletada pela entrevista. Os autores concluíram que o uso do protocolo leva à maior qualidade nas entrevistas forenses, o que é de máxima importância para a preservação dos direitos, tanto das vítimas quanto dos suspeitos.

Os estudos citados obtiveram resultados que demonstram que o Protocolo NICHD de investigação forense traz uma quantidade maior de questões abertas, diminuindo o índice de indução de questões e obtendo mais detalhes sobre a situação abusiva (Lamb et al., 2008). O instrumento tem sido um dos protocolos mais indicados por pesquisadores e contempla as diretrizes indicadas pela American Professional Society on the Abuse of Children (APSAC), organização não governamental que tem o propósito de auxiliar profissionais e serviços que trabalham com crianças vítimas de maus-tratos e suas famílias. É uma entidade reconhecida mundialmente pelas suas publicações trimestrais como o periódico Child Maltreatment e o APSAC Advisor. 
O guia da APSAC ressalta a importância das entrevistas forenses para a identificação de outras vítimas envolvidas, possibilitando profissionais a avaliarem situações de risco e proteção em crianças e suas famílias com suspeita de abuso e consequentemente auxiliando a tomada de decisão judicial (APSAC, 2012), o que é possível por meio do protocolo NICHD.

O protocolo NICHD foi adaptado para o contexto de Portugal (Peixoto, Ribeiro, \& Alberto, 2013) e no momento encontra-se em validação no Brasil por meio de pesquisas realizadas em três universidades distintas (Universidade Federal de São Carlos [UFSCar]; Universidade Tuiutí do Paraná [UTP] e Pontifícia Universidade Católica do Rio Grande do Sul [PUCRS]), localizadas em três Estados brasileiros (São Paulo, Paraná e Rio Grande do Sul). A tradução do Protocolo NICHD adaptada para o contexto brasileiro pode ser acessada em http://nichdprotocol.com/nichdbrazil.pdf (Williams, Hackbarth, Aznar-Blefari, \& Padilha, 2012).

\section{Conclusão}

O Protocolo de entrevista forense NICHD tem sido identificado na literatura como exemplo máximo da ciência aplicada (Brainerd \& Reyna, 2005; Saywitz, Lyon, \& Goodman, 2011) ao domínio da entrevista com crianças. O reconhecimento alargado reunido por este protocolo de entrevista tem sido baseado na sua forte base empírica (Lamb et al., 2008). Deve-se salientar, ainda, que a apreciação pelas entidades judiciais em diversos países tem sido positiva, sobretudo aumentando o impacto no número de casos envolvendo reconhecimento de culpa por parte do ofensor e de condenações em julgamento (Pipe et al., 2013). O protocolo tem sido utilizado em vários países, como Israel (Lamb et al., 1996), Estados Unidos da América (Lamb et al., 2008; Lamb et al., 2007), Suécia (Cederborg et al., 2000), Canada (Cyr \& Lamb, 2009), Reino Unido (Lamb et al., 2008), sendo que os resultados da sua aplicação indicam um elevado nível de replicação dos efeitos na qualidade e quantidade de informação obtida.
Há entretanto algumas limitações com o protocolo NICHD. Estudos com a utilização do mesmo (Hershkowitz et al., 2005; Hershkowitz, Fisher, et al., 2007) indicam algumas dificuldades na entrevistas de crianças relutantes em fazer revelações. Porém, tudo leva a crer que um maior investimento na relação com a criança poderá ser a chave para contornar a relutância da criança em informar (Lamb et al., 2013).

As pesquisas que estão se iniciando no Brasil com o protocolo NICHD poderão fornecer ao sistema de justiça brasileiro um instrumento adequado à entrevista com crianças em casos suspeitos de abuso sexual, garantindo uma maior eficácia e robustez da prova testemunhal. Por sua vez, espera-se subsidiar particularmente o psicólogo que atua em delegacias, em perícias ou em Varas de Família e Varas da infância e da Juventude em sua prática, fornecendo-lhe mais segurança e eficácia na entrevista, minimizando a possibilidade de contaminação do relato da criança e aumentando o seu contributo na responsabilização do agressor.

\section{Referências}

Aldridge, J., Lamb, M. E., Sternberg, K. J., Orbach, Y., Esplin, P. W., \& Bowler, L. (2004). Using a human figure drawing to elicit information from alleged victims of child sexual abuse. Journal of Consulting and Clinical Psychology in the Public Domain, 72, 304-316. doi:10.1037/0022006X.72.2.304

American Professional Society on the Abuse of Children. (2012). Practice guideline Forensic interviewing in cases of suspect child abuse. Elmhurst, IL: Author.

Anderson, J., Ellefson, J., Lashley, J., Lukas, A., Miller, S. O., Russell, A., Stauffer, J., Weigman, J. (2010). The cornerhouse forensic interview protocol: RATAC®. Thomas M. Cooley Journal of Practical \& Clinical Law, 12, 193-332.

Brainerd, C. J., \& Reyna, V. F. (2005). The science of false memory. Oxford, UK: Oxford University Press.

Brown, D. A., \& Lamb, M. E. (2009). Forensic interviews with children. A two-way street: Supporting Interviewers in adhering to best practice recommendations and enhancing children's ca- 
pabilities in forensic interviews. In K. Kuehnle \& M. Connell (Eds), The evaluation of child sexual abuse allegations. A comprehensive guide to assessment and testimony (pp. 299-309). Hoboken, NJ: Wiley.

Ceci, S. J., \& Bruck, M. (1995). Jeopardy in the courtroom: A scientific analysis of children's testimony. Washington, DC: American Psychological Association.

Cederborg, A. C., Orbach, Y., Sternberg, K. J., \& Lamb, M. E. (2000). Investigative interviews of child witnesses in Sweden. Child Abuse \& Neglect, 24(10), 1355-1361.

Cezar, J. A. D. (2007). Depoimento sem dano: Uma alternativa para inquirir crianças e adolescentes nos processos judiciais. Porto Alegre, RS: Livraria do Advogado.

Constituição da República Federativa do Brasil. (1997). Texto constitucional de 5 de outubro de 1988 (17. ed.). São Paulo, SP: Saraiva. (Original publicado em 1988)

Cronch, L. E., Viljoen, J. L., \& Hansen, D. J. (2006). Forensic interviewing in child sexual abuse cases: Current techniques and future directions. Aggression and Violent Behavior, 11, 195-207.

Cunningham, A. (2009). Dificuldades apresentadas por crianças ao prestar depoimento no Fórum Judicial e como ajudá-las (L. C. A. Williams, Trad.). In L. C. A. Williams \& E. A. C. Araújo (Eds.), Prevenção do abuso sexual infantil: Um enfoque interdisciplinar (pp. 162-167). Curitiba, PR: Juruá.

Cyr, M., Dion, J., Mcduff, P., \& Trotier-Sylvaini, K. (2012). Transfer of skills in the context of nonsuggestive investigative interviews: Impact of structured interview protocol and feedback. Applied Cognitive Psychology, 26, 516-524.

Cyr, M., \& Lamb, M. E. (2009). Assessing the effectiveness of the NICHD investigative interview protocol when interviewing French-speaking alleged victims of child sexual abuse in Quebec. Child Abuse \& Neglect, 33, 257-268.

Dobke, V. (2001). Abuso sexual: A inquirição das crianças: Uma abordagem interdisciplinar. Porto Alegre, RS: Lenz.

Estatuto da Criança e do Adolescente. (1990, 16 jul.). Lei $N^{\circ} 8.069 / 90$, de 13/07/1990. Diário Oficial da União.

Feix, L. F., \& Pergher, G. K. (2010). Memória em julgamento: Técnicas de entrevista para minimizar as falsas memórias. In L. M. Stein (Ed.), Falsas memórias: Fundamentos cientificos e suas aplicações jurídicas (pp. 209-227). Porto Alegre, RS: ArtMed

Fisher, R. P., \& Geiselman, R. E. (1992). Memoryenhancing techniques for investigating interviewing: The cognitive interview. Springfield, MA: Charles C. Thomas.

Goodman, G. S., Ogle, C., Troxel, N., Lawler, M., \& Cordon, I. (2008). Crianças vítimas no sistema judiciário: Como garantir a precisão do testemunho e evitar a revitimização. In B. R. Santos \& I. B. Gonçalves (Eds.), Depoimento sem medo (?) culturas e práticas não-revitimizantes: Uma cartografia das experiências de tomada de depoimento especial de crianças e adolescentes (pp. 21-34). São Paulo, SP: Childhood Brasil.

Goodman, G. S., \& Quas, J. A. (2008). Repeated interviews and children's memory. Current Directions in Psychological Science, 17(6), 386-390.

Harris, L. S., Goodman, G. S., Augusti, E. M., Chae, Y., \& Alley, D. (2009). Children's resistance to suggestion. In K. Kuehnle \& M. Connel (Eds.), The evaluation of child sexual abuse allegations. A comprehensive guide to assessment and testimony (pp. 182-198). Hoboken, NJ: Wiley.

Herman, S. (2009). Forensic child sexual abuse evaluations. Accuracy, ethics, and admissibility. In K. Kuehnle \& M. Connel (Eds.), The evaluation of child sexual abuse allegations. A comprehensive guide to assessment and testimony (pp. 247255). Hoboken, NJ: Wiley.

Hershkowitz, I., Fisher, S., Lamb, M. E., \& Horowitz, D. (2007). Improving credibility assessment in child sexual abuse allegations: The role of the NICHD investigative interview protocol. Child Abuse \& Neglect, 31, 99-110.

Hershkowitz, I., Horowitz, D., \& Lamb, M. E. (2005). Trends in children's disclosure of abuse in Israel: A national study. Child Abuse \& Neglect, 29, 1203-1214.

Hershkowitz, I., Horowitz, D., Lamb, M. E., Orbach, Y., \& Sternberg, K. J. (2004). Interviewing youthful suspects in alleged sex crimes: A descriptive analysis. Child Abuse \& Neglect, 28, 423-438.

Hershkowitz, I., Lanes, O., \& Lamb, M. E. (2007). Exploring the disclosure of child sexual abuse with alleged victims and their parents. Child Abuse \& Neglect, 31, 111-123. 
Hershkowitz, I., Orbach, Y., Lamb, M. E., Sternberg, K. J., \& Horowitz, D. (2006). Dynamics of forensic interviews with suspected abuse victims who do not disclose abuse. Child Abuse \& $\mathrm{Ne}$ glect, 30, 753-769.

Home Office. (1992). Memorandum of good practice on video recorded interviews with child witnesses for criminal proceedings. London: Author.

Home Office. (2011). Achieving the best evidence in criminal proceedings: Guidance for vulnerable and intimidated witnesses, including children. London: Author.

Katz, C., \& Hershkowitz, I. (2012). The effect of multipart prompts on children's testimonies in sexual abuse investigations. Child Abuse \& $\mathrm{Ne}$ glect, 36(11-12), 753-759.

Klemfuss, J. Z., \& Ceci, S. (2009). Normative memory development and the child witness. In K. Kuehnle \& M. Connel (Eds.), The evaluation of child sexual abuse allegations. A comprehensive guide to assessment and testimony (pp. 153173). Hoboken, NJ: Wiley.

La Rooy, D., Lamb, M. E., \& Pipe, M. E. (2009). Repeated interviewing. A critical evaluation of the risks and potential benefits. In K. Kuehnle \& M. Connel (Eds.), The evaluation of child sexual abuse allegations. A comprehensive guide to assessment and testimony (pp. 182-198). Hoboken, NJ: Wiley.

Lamb, M. E., \& Garretson, M. E. (2003). The effects of interviewer gender and child gender on the informativeness of alleged child sexual abuse victims in forensic interviews. Law and Human Behavior, 27, 71-157.

Lamb, M. E., Hershkowitz, I., \& Lyon, T. D. (2013). Interviewing victims and suspected victims who are reluctant to talk. APSAC Advisor, 25(4), 1619.

Lamb, M. E., Hershkowitz, I. Y., Orbach, W. E., \& Esplin, P. W. (2008). Tell me what happened: Structured investigative interviews of child victims and witnesses: Wiley Series in Psychology of Crime, Policing and Law. Chichester, UK: Wiley Blackwell.

Lamb, M. E., Hershkowitz, I., Sternberg, K. J., Esplin, P. W., Hovav, M., Manor, T., \& Yudilevitch, L. (1996). Effects of investigative utterance types on Israeli children's responses. International Journal of Behavioral Development, 19(3), 627-637.
Lamb, M. E., Orbach, Y., Hershkowitz, I., Esplin, P. W., \& Horowitz, D. (2007). A structured forensic interview protocol improves the quality and informativeness of investigative interviews with children: A review of research using the NICHD Investigative Interview Protocol. Child Abuse \& Neglect, 1201-1231.

Lamb, M. E., Sternberg, K. J., Orbach, Y., Hershkowitz, I., \& Horowitz, D. (2003) Differences between accounts provided by witnesses and alleged victims of child sexual abuse. Child Abuse \& Neglect, 27, 1019-1031.

Lyon, T. D., Lamb, M. E., \& Myers, J. (2009). Authors' response to Vieth: Legal and psychological support for the NICHD Interviewing Protocol. Child Abuse \& Neglect, 71-74.

Malloy, L. C., Brubacher, S. P., \& Lamb, M. E. (2011). Expected consequences of disclosure revealed in investigative interviews with suspected victims of child sexual abuse. Applied Developmental Science, 15, 8-19, doi:10.1080/ 10888691.2011 .538616

Memon, A., Meissner, C. A., \& Fraser, J. (2010). The cognitive interview: A meta-analytic review and study space analysis of the past 25 years. Psychology, Public Policy, and Law, 16(4), 340-372.

Olafson, E. (2012). A call for field-relevant research about child forensic interviewing for child protection. Journal of Child Sexual Abuse, 21, 109129. doi:10.1080/10538712.2012.642469

Oliveira, A. C. (2010). Abuso sexual ou alienação parental: $O$ difícil diagnóstico. Recuperado em 01 de julho, 2012, de http://igualdadeparental. blogspot.com.br/2010/11/abuso-sexual-ou-alienacao-parental-o.html\#!/2010/11/abuso-sexual-ou-alienacao-parental-o.html

Orbach, Y., Hershkowitz, I., Lamb, M. E., Sternberg, K. J., Esplin, P. W., \& Horowitz, D. (2000). Assessing the value of structured protocols for Forensic Interviews of alleged child abuse victims. Child Abuse \& Neglect, 24(6), 733-752.

Orbach, Y., \& Pipe, M. E. (2011). Investigating substantive issues. In M. E. Lamb, D. J. La Rooy, L. Malloy, \& C. Katz (Eds.), Children's testimony: $A$ handbook of psychological research and forensic practice ( $2^{\text {nd }}$ ed., pp. 147-164). Chichester, UK: Wiley-Blackwell.

Orbach, Y., Shiloach, H., \& Lamb, M. E. (2007). Reluctant disclosers of child sexual abuse. In M.E. Pipe, M. E. Lamb, Y. Orbach, \& A.-C. Ced- 
erborg (Eds.), Child sexual abuse: Disclosure, delay, and denial (pp. 115-134). New York: Routledge.

Padilha, M. G., \& Antunes, M. C. (2009). Considerações sobre depoimento sem dano em casos de abuso sexual contra crianças e adolescentes. In L. C. A. Williams \& E. C. Araújo (Eds.), Prevenção do abuso sexual infantil - Um enfoque Interdisciplinar (pp. 178-189). Curitiba, PR: Juruá.

Peixoto, C. E., Ribeiro, C., \& Alberto, I. (2013). O Protocolo de Entrevista Forense do NICHD: Contributo na obtenção do testemunho da criança no contexto português. Ministério Público, 181-219.

Peixoto, C. E., Ribeiro, C., \& Lamb, M. E. (2011). Forensic interview protocol in child sexual abuse. Why and what for? In T. Magalhães (Ed.), Abuse \& neglect series: To improve the management of child abuse and neglect (Vol. 1). Maia, Portugal: Sociedade Portuguesa para o Estudo da Criança Abusada e Negligenciada.

Peixoto, C. E., Ribeiro, C., \& Magalhães, T. (2013). Entrevista forense de crianças alegadamente vítimas de abuso. In T. Magalhães \& D. N. Vieira (Eds.), Agressões sexuais: Intervenção pericial integrada (p. 75-102). Maia: SPECAN.

Pelisoli, C., Gava, L., \& Dell'Aglio, D. (2011). Psicologia jurídica e tomada de decisão em situações envolvendo abuso sexual infantil. Psico-USF, $16(3), 327-328$.

Phillips, E., Oxburgh, G., Gavin, A., \& Myklebust, T. (2012). Investigative interviews with victims of child sexual abuse: The relationship between question type and investigation relevant information. Journal of Police and Criminal Psychology, 27, 45-54. doi:10.1007/s11896-011-9093-z

Pipe, M., Orbach, Y., Lamb, M., Abbott, C., \& Stewart, H. (2013). Do case outcomes change when investigative interviewing practices change? Psychology, Public Policy, and Law, 19(2), 179. 190.

Roberts, K. P., Brubacher, S. P., Powell, M. B., \& Price, H. L. (2011). Practice narratives. In M. E. Lamb, D. J. La Rooy, L. Malloy, \& C. Katz (Eds.), Children's testimony: A handbook of psychological research and forensic practice ( $2^{\text {nd }}$ ed., pp. 129-45). Chichester, UK: WileyBlackwell.

Salvagni, E. P., \& Wagner, M. B. (2006). Development of a Questionnaire for the Assessment of
Sexual abuse in children and estimation of its discriminant validity: A case-control study. Jornal de Pediatria, 82(6), p. 431-436.

Santos, B. R., \& Gonçalves, I. B. (Eds.). (2008). Depoimento sem medo (?) culturas e práticas não-revitimizantes: Uma cartografia das experiências de tomada de depoimento especial de crianças e adolescentes. São Paulo, SP: Childhood Brasil.

Saywitz, K., Lyon, T., \& Goodman, G. (2011). Interviewing children. In J. E. B. Myers (Ed.), The APSAC handbook on child maltreatment ( $3^{\text {rd }}$ ed., pp. 337-360). Thousand Oaks, CA: Sage.

Snider, S. M., \& Everson, M. D. (2011). What is my next question? Using question frameworks to improve children's narrative accounts of abuse. American Professional Society on the Abuse of Children, 2, 11.

Toth, P. J. D. (2011). Comparing the NICHD and RATAC Child Forensic Interview Approaches - Do the differences matter? Official News Letter of the International Society for Prevention of Child Abuse and Neglect, 20(1), 1.

Volnovich, J. (Ed.). (2005). Abuso sexual na infância. Rio de Janeiro, RJ: Lacerda.

Williams, L. C. A. (2009). Introdução ao estudo do abuso sexual infantil e análise do fenômeno no município de São Carlos. In L. C. A. Williams \& E. A. C. Araújo (Eds.), Prevenção do abuso sexual infantil: Um enfoque interdisciplinar (pp. 21-37). Curitiba, PR: Juruá.

Williams, L. C. A., Hackbarth, C., Aznar-Blefari, C., \& Padilha, M. G. S. (2012). NICHD Protocol International Evidence-Based Investigative Interviewing of Children. Retrieved from http:// nichdprotocol.com/the-nichd-protocol/

Wolfe, V. V. (1998). Child sexual abuse. In G. Mash \& R. Barkley, Treatment of childhood disorders (pp. 545-597). New York: The Guilford Press.

Yuille, J. C., Hunter, R., Joffe, R., \& Zaparniuk, J. (1993). Interviewing children in sexual abuse cases. In G. S. Goodman \& B. L. Bottoms (Eds.), Child victims, child witness: Understanding and improving testimony (pp. 95-115). New York: Guilford Press. 